\title{
Acetylcysteine for prevention of contrast-induced nephropathy after intravascular angiography: A systematic review and meta-analysis
}

\author{
Sean M Bagshaw ${ }^{1,2,3}$ and William A Ghali*2,3,4
}

\author{
Address: ${ }^{1}$ Department of Critical Care Medicine, Calgary Health Region, Calgary, Canada, ${ }^{2}$ Department of Medicine, University of Calgary, \\ Calgary, Canada, ${ }^{3}$ Department of Community Health Sciences, University of Calgary, Calgary, Canada and ${ }^{4}$ Centre for Health and Policy Studies, \\ University of Calgary, Calgary, Canada \\ Email: Sean M Bagshaw - smbagsha@ucaglary.ca; William A Ghali* - wghali@ucalgary.ca \\ * Corresponding author
}

Published: 22 October 2004

BMC Medicine 2004, 2:38 doi:10.1186/1741-70/5-2-38
Received: 16 April 2004

Accepted: 22 October 2004

This article is available from: http://www.biomedcentral.com/174I-7015/2/38

(c) 2004 Bagshaw and Ghali; licensee BioMed Central Ltd.

This is an open-access article distributed under the terms of the Creative Commons Attribution License (http://creativecommons.org/licenses/by/2.0), which permits unrestricted use, distribution, and reproduction in any medium, provided the original work is properly cited.

\begin{abstract}
Background: Contrast-induced nephropathy is an important cause of acute renal failure. We assess the efficacy of acetylcysteine for prevention of contrast-induced nephropathy among patients undergoing intravascular angiography.

Methods: We conducted a systematic review and meta-analysis of randomized controlled trials comparing prophylactic acetylcysteine plus hydration versus hydration alone in patients undergoing intravascular angiography. Studies were identified by searching MEDLINE, EMBASE, and CENTRAL databases. Our main outcome measures were the risk of contrast-induced nephropathy and the difference in serum creatinine between acetylcysteine and control groups at $48 \mathrm{~h}$.
\end{abstract}

Results: Fourteen studies involving $126 \mathrm{I}$ patients were identified and included for analysis, and findings were heterogeneous across studies. Acetylcysteine was associated with a significantly reduced incidence of contrast-induced nephropathy in five studies, and no difference in the other nine (with a trend toward a higher incidence in six of the latter studies). The pooled odds ratio for contrast-induced nephropathy with acetylcysteine relative to control was $0.54(95 \% \mathrm{Cl}, 0.32-0.91$, $P=0.02$ ) and the pooled estimate of difference in 48-h serum creatinine for acetylcysteine relative to control was $-7.2 \mu \mathrm{mol} / \mathrm{L}(95 \% \mathrm{Cl}-19.7$ to $5.3, \mathrm{p}=0.26)$. These pooled values need to be interpreted cautiously because of the heterogeneity across studies, and due to evidence of publication bias. Meta-regression suggested that the heterogeneity might be partially explained by whether the angiography was performed electively or as emergency.

Conclusion: These findings indicate that published studies of acetylcysteine for prevention of contrast-induced nephropathy yield inconsistent results. The efficacy of acetylcysteine will remain uncertain unless a large well-designed multi-center trial is performed.

\section{Background}

Contrast-induced nephropathy is a leading cause for acquired acute reductions in kidney function $[1,2]$.
Despite advances in supportive therapy, the incidence of contrast-induced nephropathy may continue to increase significantly with the broader utilization of radiocontrast 
media for diagnostic and interventional procedures.[3] Furthermore, contrast-induced nephropathy is associated with a greater risk of in-hospital morbidity, mortality, prolonged hospitalization, increased health care costs and potentially irreversible reduction in kidney function [4-8].

The pathophysiology of contrast-induced nephropathy remains incompletely understood. However, current evidence suggests that contrast media induce prolonged vasoconstriction and medullary ischemia coupled with generation of free radicals and oxidative injury to tubular cells [9-11].

Acetylcysteine, a thiol-containing anti-oxidant, has been hypothesized to prevent contrast-induced nephropathy. The potential benefit of acetylcysteine is believed to be mediated by its properties as a scavenger of free-radical species and by increasing the synthesis of nitric oxide, a potent vasodilator, in response to ischemic or other toxic injury in the kidney [12]. Given the recent publication of a series of randomized controlled trials assessing the efficacy of acetylcysteine in preventing the decline in kidney function following contrast exposure associated with intravascular angiography, we sought to conduct a systematic review and meta-analysis of these trials. The specific objectives of our meta-analysis were to assess the effect of acetycysteine on 1) the dichotomous endpoint of contrast-induced nephropathy (yes/no) and 2) serum creatinine levels following the administration of contrast media. We also conduct a meta-regression analysis to determine whether particular clinical or study quality factors influence the apparent effect of acetylcysteine on risk of contrast-induced nephropathy.

\section{Methods}

\section{Search strategy}

We identified published randomized controlled trials of acetylcysteine for prevention of contrast-induced nephropathy during intravascular angiography using both electronic and manual search strategies. We supplemented this by scanning the reference lists of all identified articles, reviewing selected conference proceedings, and by contacting experts in the field. All languages and types of publications were considered eligible. The comprehensive literature search was initially performed in April 2003 and updated in June 2004 to identify any potential new studies that may have appeared.

MEDLINE (1966 through April, 2003), EMBASE (1980 through April, 2003) and CENTRAL (Cochrane Controlled Clinical Trials Register 1996 through April, 2003) databases were searched via OVID using an approach recommended for systematic reviews of randomized trials [13]. PubMed was also searched [14]. We derived three comprehensive search themes that were then combined using the Boolean operator 'and'. The first theme used a recommended highly sensitive randomized controlled trial filter and systematic review filter method [15]. The second theme, contrast-induced nephropathy, was created by using the Boolean search term 'or' to search for the following terms appearing as both exploded medical subject headings (MeSH) or text words: 'contrast media' or 'radiocontrast' or 'kidney failure' or 'acute renal failure' or ' chronic renal failure' or 'contrast nephropathy' or 'dialysis'. The third theme, acetylcysteine, was created by a search using an exploded MeSH heading and textword search for: 'N-acetylcysteine' or 'NAC' or 'acetylcysteine' or 'Mucomyst'.

\section{Study selection criteria}

Two individuals (SMB and WAG) independently evaluated identified articles for eligibility on the basis of four inclusion criteria: 1) study design (randomized controlled trials), 2) target population (patients undergoing intravascular angiography), 3) intervention (trials of acetylcysteine plus hydration versus control) and 4) outcome (trials with explicit definition of contrast-induced nephropathy).

\section{Data extraction}

Two reviewers (SMB and WAG) independently extracted data from all primary studies fulfilling eligibility criteria. Any discrepancies in extracted data were resolved by consensus. Data extracted included identifying information, focus of the study, details of study protocol and demographic data. The primary outcome measures were the incidence of contrast-induced nephropathy and change in serum creatinine. The secondary outcome measure was requirement for renal replacement therapy. Authors of the studies were contacted for additional information when applicable.

\section{Assessment of methodological quality}

Two reviewers (SMB and WAG) independently assessed methodological quality of individual studies. Any disagreements were resolved by consensus. Items used to assess study quality were methods of randomization, any blinding, use of a placebo, reporting of losses to follow-up or missing outcome assessments, and evidence of important baseline differences between the groups [16-18]. An overall quality score was determined for each study as described by Jadad et al [16].

\section{Prior hypotheses regarding sources of heterogeneity}

The presence of heterogeneity can compromise the interpretation and validity of meta-analyses and can result from significant differences in methodology, study populations, interventions, outcomes, or chance [19]. A priori consideration of potential factors contributing to heterogeneity for acetylcysteine in prevention of contrast- 
induced nephropathy included baseline serum creatinine levels, volume of contrast media, volume of hydration, age, diabetes mellitus, elective or emergency procedure, and a number of trial methodology factors.

\section{Statistical methods}

Data from all of the selected randomized controlled trials were combined to estimate the pooled odds ratio (OR) with 95\% confidence intervals (CIs) using a randomeffects model as described by Der Simonian and Laird $[20,21]$. The presence of heterogeneity across trials was evaluated using a chi-square test for homogeneity [22]. Meta-regression was performed to analyze for potential clinical and study quality factors that may influence treatment effects. We tested for potential publication bias using both a Begg's test for asymmetry and an Egger's test $[23,24]$. All statistical analyses were performed with Stata version 8.0 (StataCorp, College Station, TX).

\section{Results}

\section{Identification of studies}

A total of 66 unique citations were identified by our initial search strategy (Figure 1). After the initial screen, 22 citations warranted further review. Among these, 15 citations were excluded: 8 were clinical reviews, 3 were prospective cohort studies, 2 were substudies of previously published randomized controlled trials, one did not include a control group, and one did not involve intravascular angiography. Therefore, we had identified 7 studies for inclusion. A repeat search of the literature conducted in June 2004 yielded seven additional eligible studies. Overall, 14 studies thus fulfilled our inclusion criteria [25-38]. All of these citations were identified by the electronic search strategy and are published in peer-reviewed journals [39].

\section{Study characteristics}

All the randomized controlled trials were published in the years 2002 through 2004. Tables 1 and 2 present the characteristics of the 14 randomized controlled trials. A total of 1261 patients were studied in these 14 randomized controlled trials, among whom 631 received acetylcysteine and 630 were in control groups. There were 563 $(44.6 \%)$ patients with diabetes mellitus, of whom 284 were assigned to receive acetylcysteine and 279 were assigned to a control group. The dosing and schedule of administration of acetylcysteine was variable across studies; however, in the majority of studies, acetylcysteine was initiated $12-24 \mathrm{~h}$ prior to angiography. In two trials, large doses of acetylcysteine were administered immediately prior to (within $1 \mathrm{~h}$ ) and shortly following (within 3-4 h) angiography $[26,29]$. All patients were administered a hydration protocol around their procedure and all received low or iso-osmolar non-ionic contrast media.
The definition of contrast-induced nephropathy was variable across studies. Four studies defined contrast-induced nephropathy as a $>44.2 \mu \mathrm{mol} / \mathrm{L}$ increase in serum creatinine from baseline $[25,29,32,37]$, four used a $>25 \%$ increase in serum creatinine from baseline $[26,30,33,35]$, four used either $\mathrm{a}>44.2 \mu \mathrm{mol} / \mathrm{L}$ or $\mathrm{a}>25 \%$ increase in serum creatinine from baseline $[28,31,34,36]$, one used either a $>44.2 \mu \mathrm{mol} / \mathrm{L}$ or a $>33 \%$ increase in serum creatinine from baseline[38] and one study combined either a $>25 \%$ increase in serum creatinine from baseline or dialysis [27]. Generally, the time for ascertaining contrastinduced nephropathy for all studies was $48 \mathrm{~h}$ after the exposure to contrast media, with the exception of four studies, where presence or absence of contrast-induced nephropathy was determined at 24,72 and $96 \mathrm{~h}$ $[26,30,34,35]$.

\section{Meta-analysis of incidence of contrast-induced nephropathy}

The reported incidence of contrast-induced nephropathy was variable across studies. Table 3 and Figure 2 present information on the incidence of contrast-induced nephropathy for all studies. Five studies provided evidence of a risk reduction for development of contrast-induced nephropathy with acetylcysteine $[26,28,33,35,37]$, whereas nine studies reported no evidence of benefit $[25,27,29$ $32,34,36,38]$. Furthermore, six of the latter studies yielded an odds ratio $>1.0$, suggesting a trend towards an increased risk of contrast-induced nephropathy $[25,29,31,32,36,38]$. The overall pooled odds ratio for development of contrast-induced nephropathy using a random-effects model was 0.54 (95\% CI, 0.32-0.91, p = 0.022 ), suggesting a significant reduction in CIN with acetylcysteine (Figure 2). However, this pooled odds ratio should be interpreted with caution because the analysis comparing the occurrence of contrast-induced nephropathy across all studies revealed significant heterogeneity (chi-square $=23.96, p=0.032$ ). In total, six patients required dialysis, among whom two received acetylcysteine and two were in control groups. Group assignment was not reported for the other two patients who required dialysis.

\section{Meta-analysis of change in serum creatinine with acetylcysteine}

Table 3 shows a summary of the changes in serum creatinine across studies. The pooled estimate (using a random effects model) for the difference in $48 \mathrm{~h}$ serum creatinine between the acetylcysteine and control groups was -7.2 $\mu \mathrm{mol} / \mathrm{L}(95 \%$ CI -19.7 to $5.3, \mathrm{p}=0.26$ ) based on data available from eight studies $[25,27,28,31,32,35,37,38]$. This suggests no significant absolute change in serum creatinine with the administration of acetylcysteine (Figure 3 ). Again, this pooled estimate requires cautious interpretation owing to the availability of data from only eight 


\section{Citations screened}

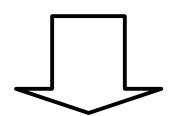

22 Potentially relevant citations identified for further review

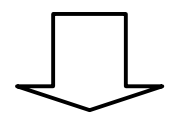

15 Articles Excluded: 8 Reviews

3 Prospective cohort studies

2 Substudies of RCTs

1 No control group

1 Not angiography

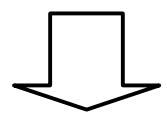

7 Randomized controlled trials initially included

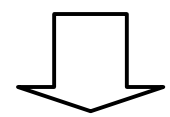

7 Additional randomized controlled trials included from follow-up verification search

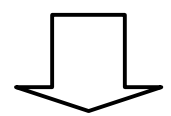

14 Randomized controlled trials included in final analysis

Figure I

Flow diagram of study selection process. 
Table I: Characteristics of studies reporting on the use of acetylcysteine for prevention of contrast-induced nephropathy.

\begin{tabular}{|c|c|c|c|c|c|c|c|c|c|}
\hline \multirow[t]{2}{*}{ First Author } & \multirow[t]{2}{*}{ Patients } & \multirow[t]{2}{*}{ Diabetes } & \multirow[t]{2}{*}{$\begin{array}{c}\text { Elective } \\
\text { Procedure }\end{array}$} & \multirow[t]{2}{*}{$\begin{array}{l}\text { Primary } \\
\text { Outcome }\end{array}$} & \multirow[t]{2}{*}{$\begin{array}{l}\text { Acetylcysteine } \\
\text { Protocol* }\end{array}$} & \multirow[t]{2}{*}{$\begin{array}{c}\text { Hydration } \\
\text { Protocol }\end{array}$} & \multirow[t]{2}{*}{$\begin{array}{l}\text { Contrast } \\
\text { Media }\end{array}$} & \multicolumn{2}{|c|}{$\begin{array}{l}\text { Contrast Media } \\
\text { Volume }(\mathrm{mL})^{* *}\end{array}$} \\
\hline & & & & & & & & NAC & Control \\
\hline $\begin{array}{l}\text { Allaqaband, } \\
2002^{25}\end{array}$ & 85 & $4 \mid(48 \%)$ & Unclear & $\begin{array}{c}>44.2 \mu \mathrm{mol} / \mathrm{L} \\
\text { increase SCr } \\
\text { at } 48 \mathrm{hrs}\end{array}$ & $\begin{array}{c}600 \mathrm{mg} \text { PO bid } \\
\text { pre/post }\end{array}$ & $\begin{array}{c}0.45 \% \mathrm{NS} I \mathrm{~mL} / \\
\mathrm{kg} / \mathrm{hr} I 2 \mathrm{hr} \text { pre/ } \\
\text { post }\end{array}$ & $\begin{array}{l}\text { loversol or } \\
\text { lodixanol }\end{array}$ & 121.6 & 122 \\
\hline $\begin{array}{l}\text { Baker, } \\
2003^{26}\end{array}$ & 80 & 34 (43\%) & Unclear & $\begin{array}{c}>25 \% \\
\text { increase } \mathrm{SCr} \\
\text { at } 48 \text { or } 96 \\
\text { hrs }\end{array}$ & $\begin{array}{l}150 \mathrm{mg} / \mathrm{kg} \text { IV } 30 \\
\mathrm{~min} \text { pre \& } 50 \mathrm{mg} / \\
\mathrm{kg} \text { IV infusion } 4 \\
\text { hrs post }\end{array}$ & $\begin{array}{l}\text { Control } 0.9 \% \mathrm{NS} \\
\mathrm{I} \mathrm{mL} / \mathrm{kg} / \mathrm{hr} 12 \mathrm{hr} \\
\text { pre/post }\end{array}$ & lodixanol & 238 & 222 \\
\hline $\begin{array}{l}\text { Briguori, } \\
2002^{27}\end{array}$ & 183 & 69 (38\%) & Yes & $\begin{array}{l}>25 \% \\
\text { increase SCr } \\
\text { at } 48 \mathrm{hrs} \text { or } \\
\text { dialysis }\end{array}$ & $\begin{array}{l}600 \text { mg PO bid } \\
\text { pre/post }\end{array}$ & $\begin{array}{c}0.45 \% \mathrm{NS} \mathrm{I} \mathrm{mL/} \\
\mathrm{kg} / \mathrm{hr} 12 \mathrm{hr} \text { pre/ } \\
\text { post }\end{array}$ & lopromide & 194 & 200 \\
\hline $\begin{array}{l}\text { Diaz- } \\
\text { Sandoval, } \\
2002^{28}\end{array}$ & 54 & 21 (39\%) & Yes & $\begin{array}{c}>44.2 \mu \mathrm{mol} / \mathrm{L} \\
\text { or } 25 \% \mathrm{SCr} \\
\text { increase at } 48 \\
\text { hrs }\end{array}$ & $\begin{array}{l}600 \text { mg PO bid } \\
\text { pre/post }\end{array}$ & $\begin{array}{c}0.45 \% \mathrm{NS} I \mathrm{~mL} / \\
\mathrm{kg} / \mathrm{hr} 2-12 \mathrm{hr} \text { pre } \\
\& 12 \mathrm{hr} \text { post }\end{array}$ & loxilan & 179 & 189 \\
\hline $\begin{array}{l}\text { Durham, } \\
2002^{29}\end{array}$ & 79 & 38 (48\%) & No & $\begin{array}{c}>44.2 \mu \mathrm{mol} / \mathrm{L} \\
\text { increase SCr } \\
\text { at } 48 \mathrm{hrs}\end{array}$ & $\begin{array}{l}1200 \mathrm{mg} \mathrm{PO} \mathrm{I} \mathrm{hr} \\
\text { pre and } 3 \mathrm{hr} \text { post }\end{array}$ & $\begin{array}{c}0.45 \% \mathrm{NS} \mathrm{I} \mathrm{mL/} \\
\mathrm{kg} / \mathrm{hr} I 2 \mathrm{hr} \text { pre/ } \\
\text { post }\end{array}$ & lohexol & 77.4 & 84.7 \\
\hline $\begin{array}{l}\text { Efrati, } \\
2003^{30}\end{array}$ & 49 & $26(53 \%)$ & Yes & $\begin{array}{c}>25 \% \text { increase } \\
\mathrm{SCr} \text { at } 24 \text { or } \\
96 \mathrm{hrs}\end{array}$ & $\begin{array}{l}1000 \mathrm{mg} \text { PO bid } \\
\text { day pre/post }\end{array}$ & $\begin{array}{c}0.45 \% \mathrm{NS} I \mathrm{~mL} / \\
\mathrm{kg} / \mathrm{hr} I 2 \mathrm{hr} \text { pre/ } \\
12 \text { post }\end{array}$ & lopromide & 142 & 138 \\
\hline Fung, 200431 & 91 & 48 (53\%) & Yes & $\begin{array}{c}>44.2 \mu \mathrm{mol} / \mathrm{L} \\
\text { or } 25 \% \\
\text { decrease in } \\
\text { GFR }\end{array}$ & $\begin{array}{l}400 \mathrm{mg} \text { PO tid } \\
\text { day prior/post }\end{array}$ & $\begin{array}{c}0.9 \% \mathrm{NS} 100 \mathrm{ml} / \\
\mathrm{hr} 12 \mathrm{hr} \text { pre } / 12 \\
\text { post }\end{array}$ & lopromide & 135.8 & 121 \\
\hline $\begin{array}{l}\text { Goldenberg, } \\
2004^{32}\end{array}$ & 80 & 43 (54\%) & No & $>44.2 \mu \mathrm{mol} / \mathrm{L}$ & $\begin{array}{l}600 \mathrm{mg} \text { PO bid } \\
\text { day prior/post }\end{array}$ & $\begin{array}{c}0.45 \% \mathrm{NS} \mathrm{I} \mathrm{mL/} \\
\mathrm{kg} / \mathrm{hr} I 2 \mathrm{hr} \text { pre/ } \\
\text { I2 post }\end{array}$ & lopamidol & 111 & 138 \\
\hline Kay, $2003^{33}$ & 200 & 75 (38\%) & Yes & $\begin{array}{c}>25 \% \\
\text { increase SCr } \\
\text { at } 48 \mathrm{hrs}\end{array}$ & $\begin{array}{c}600 \text { mg PO bid x } \\
4 \text { (3 pre) }\end{array}$ & $\begin{array}{c}0.9 \% \mathrm{NS} I \mathrm{~mL} / \mathrm{kg} / \\
\mathrm{hr} 12 \mathrm{hr} \text { pre } \& 6 \\
\mathrm{hr} \text { post }\end{array}$ & lopamidol & 130 & 120 \\
\hline $\begin{array}{l}\text { Kefer, } \\
2003^{34}\end{array}$ & 104 & $13(13 \%)$ & Unclear & $\begin{array}{c}>44.2 \mu \mathrm{mol} / \mathrm{L} \\
\text { increase SCr } \\
\text { at } 24 \mathrm{hrs}\end{array}$ & $\begin{array}{l}\text { I } 200 \mathrm{mg} \text { IV I } 2 \mathrm{hr} \\
\text { pre \& } \\
\text { immediately post }\end{array}$ & $\begin{array}{c}\text { D5W } 20 \mathrm{~mL} / \mathrm{hr} \\
12 \mathrm{hr} \text { pre \& } 24 \mathrm{hr} \\
\text { post }\end{array}$ & $\begin{array}{l}\text { lopromide } \\
\text { or lohexol }\end{array}$ & NR & NR \\
\hline $\begin{array}{l}\text { MacNeill, } \\
2003^{35}\end{array}$ & 43 & $20(46 \%)$ & Yes & $\begin{array}{c}>25 \% \\
\text { increase } \mathrm{SCr} \\
\text { at } 72 \mathrm{hrs}\end{array}$ & $\begin{array}{c}600 \mathrm{mg} \text { PO } \times 5 \\
(2 \text { pre })\end{array}$ & $\begin{array}{l}\text { Inpatient: } \\
0.45 \% \mathrm{NS} \mathrm{I} \mathrm{mL/} \\
\mathrm{kg} / \mathrm{hr} 12 \mathrm{hr} \text { pre } \\
\text { Outpatient: } \\
0.45 \% \mathrm{NS} 2 \mathrm{~mL} / \\
\mathrm{kg} / \mathrm{hr} 4 \mathrm{hr} \text { pre \& } \\
\text { both } 12 \mathrm{hr} \text { post }\end{array}$ & $\begin{array}{l}\text { lopromide } \\
\text { or loxilan }\end{array}$ & 103 & 116 \\
\hline $\begin{array}{l}\text { Oldemeyer, } \\
2003^{36}\end{array}$ & 96 & $43(45 \%)$ & Yes & $\begin{array}{c}>44.2 \mu \mathrm{mol} / \mathrm{L} \\
\text { or } 25 \% \mathrm{SCr} \\
\text { increase at } 48 \\
\text { hrs }\end{array}$ & $\begin{array}{c}1500 \mathrm{mg} \text { bid } \times 4 \\
(\text { I pre })\end{array}$ & $\begin{array}{c}0.45 \% \mathrm{NS} I \mathrm{~mL} / \\
\mathrm{kg} / \mathrm{hr} I 2 \mathrm{hr} \text { pre/ } \\
\text { post }\end{array}$ & lopamidol & 134 & 127 \\
\hline Shyu, 2002 & 121 & 77 (64\%) & Yes & $\begin{array}{c}>44.2 \mu \mathrm{mol} / \mathrm{L} \\
\text { increase SCr } \\
\text { at } 48 \mathrm{hrs}\end{array}$ & $\begin{array}{l}400 \mathrm{mg} \mathrm{PO} \text { bid } \\
\text { pre/post }\end{array}$ & $\begin{array}{c}0.45 \% \mathrm{NS} I \mathrm{~mL} / \\
\mathrm{kg} / \mathrm{hr} I 2 \mathrm{hr} \text { pre/ } \\
\text { post }\end{array}$ & lopamidol & 119 & 115 \\
\hline $\begin{array}{l}\text { Vallero, } \\
2002^{38}\end{array}$ & 100 & $23(23 \%)$ & Unclear & $\begin{array}{c}>44.2 \mu \mathrm{mol} / \mathrm{L} \\
\text { or } 33 \% \\
\text { increase SCr } \\
\text { at } 48 \mathrm{hrs}\end{array}$ & $\begin{array}{c}600 \mathrm{mg} \text { PO bid } \\
\text { pre/post }\end{array}$ & $\begin{array}{c}0.45 \% \mathrm{NS} \mathrm{I} \mathrm{mL/} \\
\mathrm{kg} / \mathrm{hr} \mathrm{I-2} \mathrm{hr} \mathrm{pre} \\
\& 24 \mathrm{hr} \text { post }\end{array}$ & lodixanol & 187.8 & 219 \\
\hline
\end{tabular}

Legend: $\mathrm{NR}=$ not recorded or available; $\mathrm{NAC}=$ acetycysteine; $\mathrm{SCr}=$ serum creatinine (for conversion to mg/dL divide by 88.4 ). $* \mathrm{NAC}$ administered with hydration protocol. **Data presented as means. 
Table 2: Summary of quality indicators for studies of acetylcysteine for prevention of contrast-induced nephropathy.

\begin{tabular}{|c|c|c|c|c|c|c|c|c|c|}
\hline First Author & $\begin{array}{l}\text { Jadad } \\
\text { score }\end{array}$ & $\begin{array}{l}\text { Inclusion/ } \\
\text { exclusion } \\
\text { criteria } \\
\text { specified }\end{array}$ & $\begin{array}{c}\text { Randomi- } \\
\text { zation } \\
\text { process } \\
\text { described }\end{array}$ & $\begin{array}{c}\text { Use of any } \\
\text { blinding }\end{array}$ & $\begin{array}{l}\text { Placebo- } \\
\text { controlled }\end{array}$ & $\begin{array}{l}\text { Reported } \\
\text { loss to } \\
\text { follow-up }\end{array}$ & $\begin{array}{l}\text { Intention- } \\
\text { to treat } \\
\text { analysis }\end{array}$ & $\begin{array}{c}\text { Potential } \\
\text { important } \\
\text { baseline } \\
\text { differences }\end{array}$ & $\begin{array}{c}\text { Power } \\
\text { calculation }\end{array}$ \\
\hline Allaqaband 25 & 3 & yes/no & yes & yes & yes & no & no & no & yes \\
\hline Baker $^{26}$ & 2 & yes/yes & yes & no & yes & no & yes & no & yes \\
\hline Briguori $^{27}$ & 1 & yes/no & yes & no & no & no & unclear & yes & no \\
\hline Diaz-Sandoval $^{28}$ & 4 & yes/yes & yes & yes & no & yes & unclear & yes & no \\
\hline Durham $^{29}$ & 5 & yes/yes & yes & yes & yes & yes & unclear & no & yes \\
\hline Efrati $^{30}$ & 4 & yes/yes & no & yes & yes & yes & unclear & no & no \\
\hline Fung ${ }^{31}$ & 4 & yes/yes & yes & no & no & no & yes & no & yes \\
\hline Goldenberg 32 & 5 & yes/yes & yes & yes & yes & no & unclear & no & yes \\
\hline Kay $^{33}$ & 5 & yes/yes & yes & yes & yes & yes & yes & no & yes \\
\hline Kefer $^{34}$ & 4 & yes/yes & yes & yes & yes & yes & unclear & yes & no \\
\hline MacNeill35 & 4 & yes/yes & yes & no & yes & no & unclear & no & yes \\
\hline Oldemeyer 36 & 4 & yes/yes & yes & yes & no & yes & unclear & no & no \\
\hline Shyu ${ }^{37}$ & 3 & yes/yes & yes & yes & no & yes & unclear & no & no \\
\hline Vallero ${ }^{38}$ & 2 & yes/yes & yes & no & no & no & unclear & yes & no \\
\hline
\end{tabular}

Legend: $\mathrm{NR}=$ not recorded or available; Jadad score range $0-5$.

Table 3: Summary of outcomes of studies of acetylcysteine for prevention of contrast-induced nephropathy.

\begin{tabular}{|c|c|c|c|c|c|c|c|c|}
\hline \multirow[t]{2}{*}{ First Author } & \multicolumn{2}{|c|}{$\begin{array}{l}\text { Contrast-induced } \\
\text { nephropathy* }\end{array}$} & \multicolumn{2}{|c|}{$\begin{array}{l}\text { Acetylcysteine Serum } \\
\text { Creatinine }(\mu \mathrm{mol} / \mathrm{L})\end{array}$} & \multicolumn{2}{|c|}{$\begin{array}{c}\text { Control Serum } \\
\text { Creatinine }(\mu \mathrm{mol} / \mathrm{L})\end{array}$} & \multicolumn{2}{|c|}{ Dialysis (N) } \\
\hline & Acetylcysteine & Control & Baseline & $\begin{array}{l}\text { Second } \\
\text { SCr }\end{array}$ & Baseline & $\begin{array}{l}\text { Second } \\
\text { SCr }\end{array}$ & NAC & Control \\
\hline Allaqaband ${ }^{25}$ & $8 / 45$ (18\%) & $6 / 40$ (15\%) & 194.5 & 196.3 & 179.5 & 179.5 & 2 & 0 \\
\hline Baker $^{26}$ & $2 / 4 \mid(5 \%)$ & $8 / 39(21 \%)$ & 163.6 & 156.5 & 154.7 & 159.1 & 0 & 0 \\
\hline Briguorii27 $^{27}$ & $6 / 92(7 \%)$ & $10 / 91$ (11\%) & 134.4 & 130.8 & 136.1 & 135.3 & 0 & I \\
\hline Diaz-Sandova|28 & $2 / 25(8 \%)$ & $13 / 29$ (45\%) & 146.7 & 135.5 & 137.9 & 166.2 & 0 & 0 \\
\hline Durham $^{29}$ & $10 / 38(26 \%)$ & $9 / 4 \mid(22 \%)$ & 194.5 & NR & 203.3 & NR & NR & NR \\
\hline Efrati ${ }^{30}$ & $0 / 24(0 \%)$ & $2 / 25(8 \%)$ & 135.3 & 143.2 & 131.7 & 143.2 & 0 & 0 \\
\hline Fung 31 & $8 / 46(17 \%)$ & $6 / 45(13 \%)$ & 200.7 & 216.6 & 209.5 & 212.2 & NR & NR \\
\hline Goldenberg 32 & $4 / 41(10 \%)$ & $3 / 39(8 \%)$ & 176.8 & 176.8 & 168.0 & 165.3 & 0 & 0 \\
\hline Kay $^{33}$ & $4 / 102(4 \%)$ & $12 / 98(12 \%)$ & 119.3 & 107.8 & 120.2 & 122.0 & 0 & 0 \\
\hline Kefer ${ }^{34}$ & $2 / 53(8 \%)$ & $3 / 51(6 \%)$ & 91.9 & 91.1 & 102.5 & 93.7 & 0 & 0 \\
\hline MacNeill35 & $|/ 2|(5 \%)$ & $7 / 22$ (32\%) & 167.1 & 168.0 & 168.0 & 210.4 & NR & $N R$ \\
\hline Oldemeyer ${ }^{36}$ & $4 / 49(8 \%)$ & $3 / 47(6 \%)$ & 144.1 & NR & 146.7 & NR & 0 & 0 \\
\hline Shyu ${ }^{37}$ & $2 / 60(3 \%)$ & $15 / 6 \mid(25 \%)$ & 247.5 & 221.0 & 247.5 & 274.0 & 0 & 1 \\
\hline Vallero $^{38}$ & $4 / 47(9 \%)$ & $4 / 53(8 \%)$ & 87.5 & 93.7 & 84 & 86.6 & NR & NR \\
\hline
\end{tabular}

Legend: $\mathrm{SCr}=$ serum creatinine (for conversion to $\mathrm{mg} / \mathrm{dL}$ divide by 88.4 ); $\mathrm{NR}=$ not recorded or available. *Values are numbers of patients with contrast-induced nephropathy/total number of patients in treatment group (\%).

studies and to the presence of significant heterogeneity across studies $(\mathrm{Q}=50.9, \mathrm{p}<0.0005)$. The change in serum creatinine at $96 \mathrm{~h}$ was assessed in two studies as a primary outcome $[26,30]$. The pooled estimate for the difference in 96-h serum creatinine for these two studies was simi- larly non-significant $[-1.8 \mu \mathrm{mol} / \mathrm{L}(95 \% \mathrm{CI}-8.9$ to $5.2, \mathrm{p}=$ $0.61)]$.

\section{Meta-regression}

Meta-regression was performed to assess a number of clinical and study quality factors that may have led to hetero- 


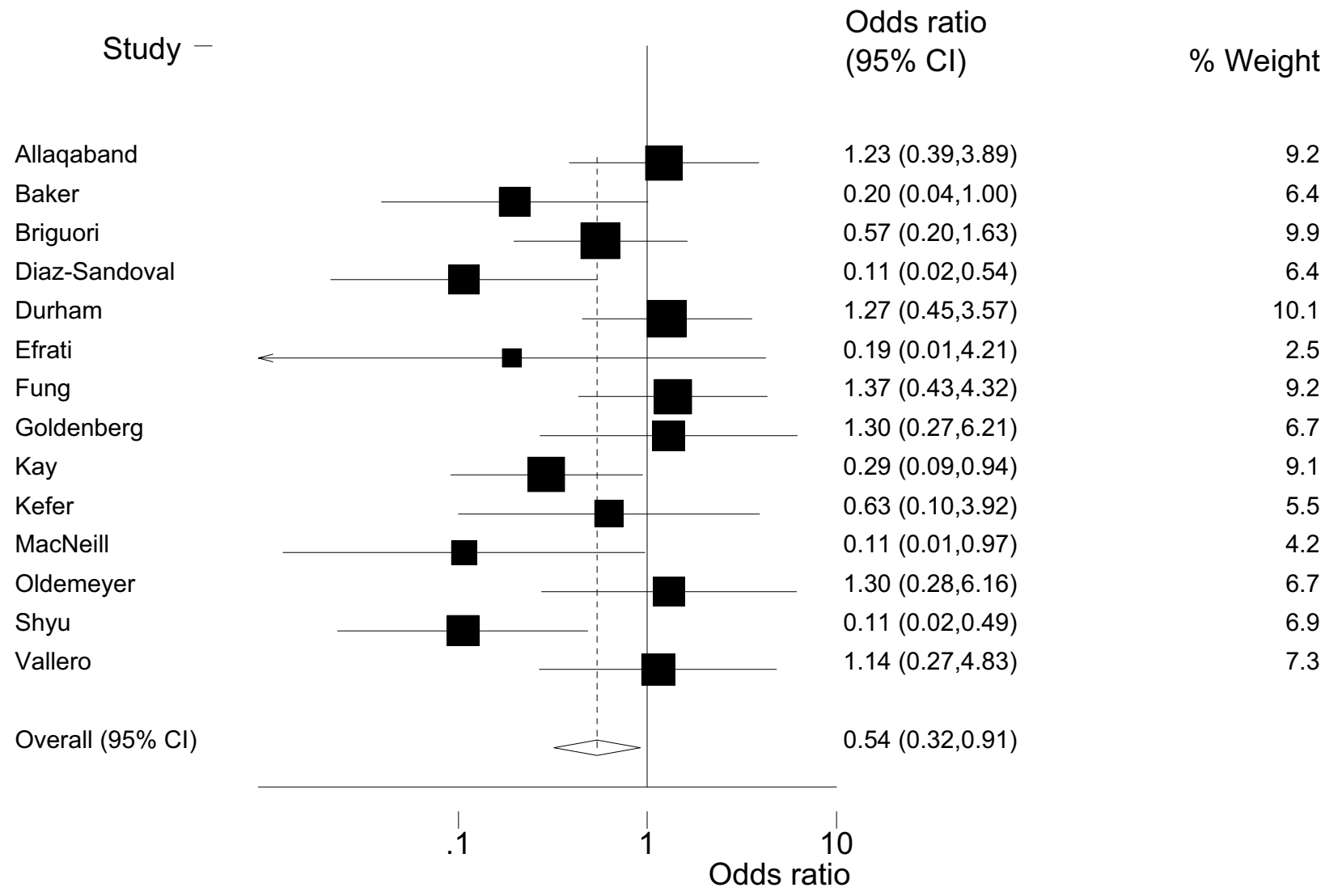

Figure 2

Forest plot of odds ratios for development of contrast-induced nephropathy from 14 trials.

geneity across studies. Interestingly, these analyses suggest that the heterogeneity may be partially explained by whether the angiography procedures were performed electively or as emergency, because studies where all enrolled patients were undergoing elective procedures had significantly lower odds ratios than did studies where emergency cases were included (coefficient for "elective-only" studies, $-0.6,95 \% \mathrm{CI},-1.24$ to $0.03, \mathrm{p}=0.06$ ).

Other meta-regression analyses demonstrated that the heterogeneity could not be accounted for by differences in patient age (coefficient $-0.04,95 \% \mathrm{CI},-0.2$ to $0.1, \mathrm{p}=0.6$ ), baseline serum creatinine (coefficient -0.001, 95\% CI, 0.01 to $0.01, \mathrm{p}=0.9)$, volume of contrast media $(-0.006$, $95 \% \mathrm{CI},-0.02$ to $0.07, \mathrm{p}=0.4$ ) or diabetes mellitus (coefficient $-0.01,95 \% \mathrm{CI},-0.03$ to 0.02 , p = 0.6). Likewise, heterogeneity was not accounted for by differences in study quality including use of blinding (coefficient $0.6,95 \% \mathrm{CI},-1.7$ to $0.5, \mathrm{p}=0.3$ ), concealment of randomization (coefficient $-0.8,95 \% \mathrm{CI},-3.8$ to $2.1, \mathrm{p}=0.6$ ), use of placebo (coefficient $-0.6,95 \% \mathrm{CI},-1.7$ to $0.5, \mathrm{p}=$ 0.30 , consecutive patient enrollment (coefficient 0.5, 95\% CI, -1.5 to $2.4, \mathrm{p}=0.6$ ) or overall Jadad score (coefficient $0.05,95 \% \mathrm{CI},-0.4$ to $0.5, \mathrm{p}=0.8$ ).

There was some evidence to suggest possible publication bias according to Begg's test ( $\mathrm{p}=0.03$, with continuity correction) and a trend with Egger's test (coefficient -3.03, $95 \% \mathrm{CI},-6.71$ to $0.65, \mathrm{p}=0.09$ ). Figure 4 demonstrates this graphically, as there is asymmetry in the funnel plot with a predominance of studies with large standard errors (i.e., usually small studies) showing benefit associated with acetylcysteine and a paucity of small negative studies.

\section{Discussion}

Our meta-analysis of 14 peer-reviewed studies of patients undergoing intravascular angiography may lead some to conclude that the administration of acetylcysteine causes a reduced incidence of contrast-induced nephropathy. However, such a conclusion may be premature based on 


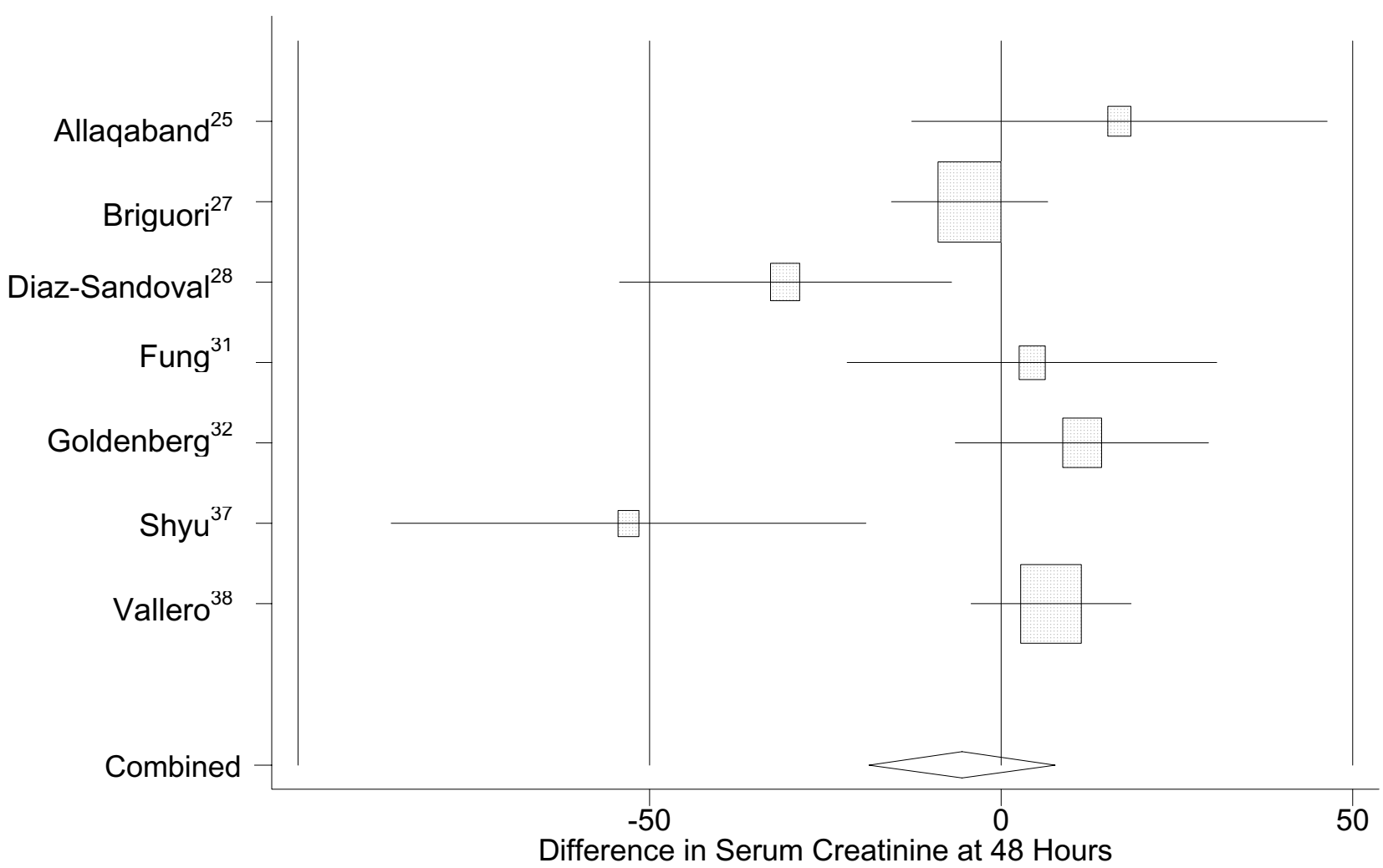

Figure 3

Forest plot of differences in serum creatinine between acetylcysteine and control at $48 \mathrm{~h}$ after contrast media administration from eight trials.

data published to date because our systematic review reveals considerable heterogeneity of findings across trials. Furthermore, our meta-analysis of post-treatment creatinine values does not reveal any truly meaningful difference in serum creatinine levels at $48 \mathrm{~h}$ between the acetylcysteine and control groups. Finally, insufficient data are available to allow inferences to be drawn about the efficacy of acetylcysteine on clinically meaningful endpoints such as dialysis, length of hospitalization or mortality.

This meta-analysis has several features that distinguish it from a similar meta-analysis by Birck et al that recently received considerable attention, and that rather firmly concluded that acetylcysteine is beneficial [40]. First, though our meta-analysis yielded a similar overall reduction in the incidence of contrast-induced nephropathy, we have included seven additional studies. Second, we have focused primarily on patients undergoing intravascular angiography. Third, we have used the pooled odds ratio across studies as a summary statistic because of its theoretical advantage to the use of relative risks in meta-analysis [21]. Fourth, we have included an analysis of differences in serum creatinine to complement the dichotomous endpoint of contrast-induced nephropathy. Fifth, we pointedly draw attention to the fact that there is some evidence to suggest publication bias, or at the very least funnel plot asymmetry. And finally, perhaps most importantly, we have explored the heterogeneity in results across studies in much greater detail than do Birck et al, and more directly address the relevance of this heterogeneity in the overall interpretation of study results. Two other meta-analyses have also recently been published, and similarly concluded that acetylcysteine is beneficial; however, these studies also failed to adequately address the issue of the considerable heterogeneity across studies $[41,42]$. Collectively, these three previously published meta-analyses unfortunately send a misleading bottomline message to the medical community - that the evidence in favor of acetylcysteine is firm [43]. Many will cor- 


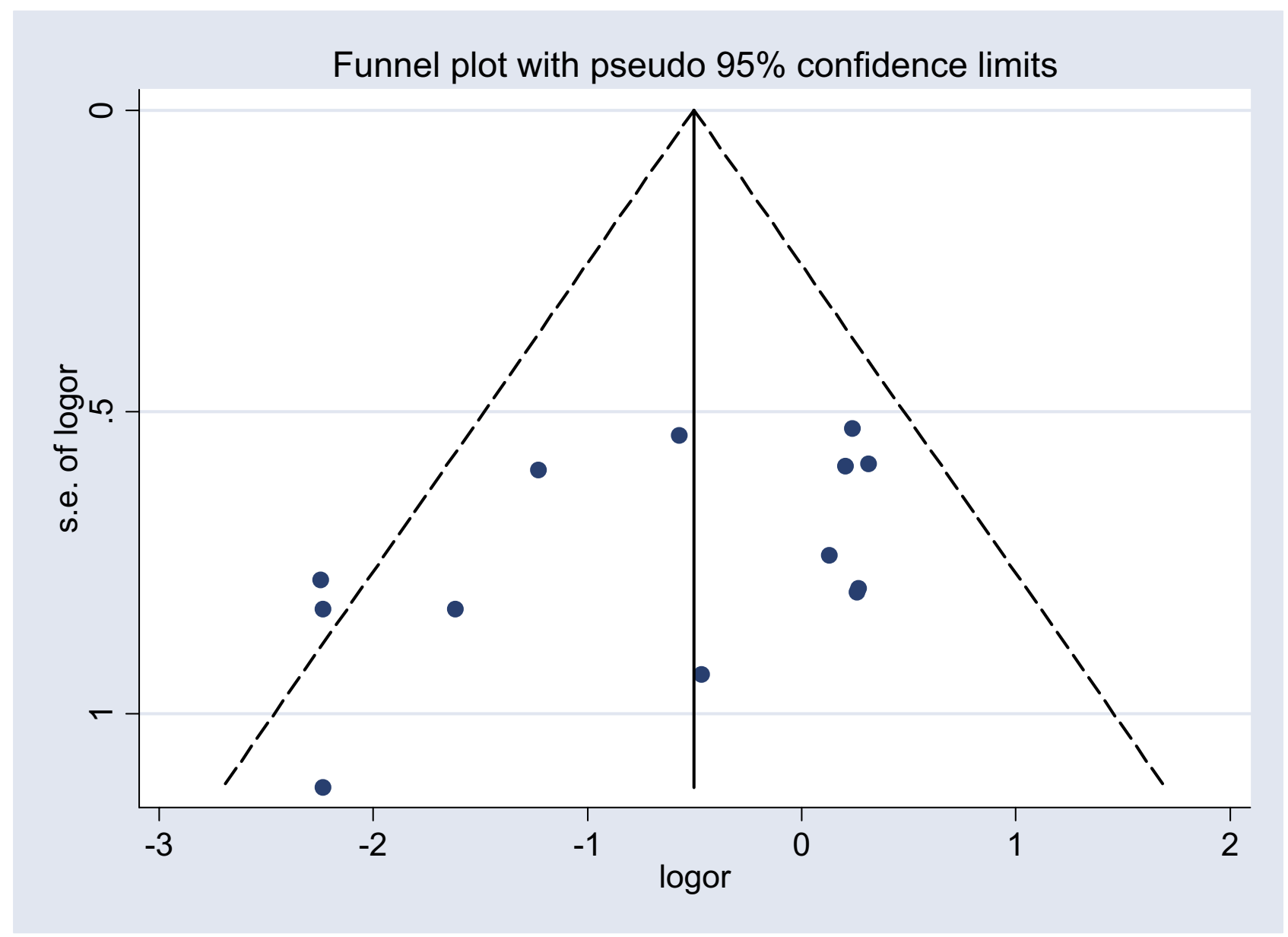

\section{Figure 4}

Evidence of publication bias by Funnel plot. Funnel plot asymmetry is demonstrated by evidence of a cluster of small studies with low-protective odds ratio and the paucity of small negative studies in the lower right of the funnel plot.

respondingly infer from these three 'positive' metaanalyses that there is no longer a need for primary research into the efficacy of acetylcysteine. Our global conclusion, meanwhile, is rather different, in that we more cautiously conclude that further data may be needed before a firm conclusion can be made regarding the efficacy of acetylcysteine. Two other very recent meta-analyses $[44,45]$ make a similar conclusion to ours, though those meta-analyses do not include as many peerreviewed and published studies as does our updated systematic review.

The presence of heterogeneity and/or publication bias can compromise the interpretation of meta-analyses and result in erroneous and potentially misleading conclusions $[19,43]$. A striking example of early meta-analysis producing misleading results is that of intravenous magnesium in the treatment of acute myocardial infarction. The results of two meta-analyses of several small clinical trials on this treatment suggested a reduction in arrhythmias and mortality $[46,47]$. Furthermore, an argument was made at the time for the use of magnesium therapy because of ease of use, favorable side effect profile and low cost $[47,48]$. However, the subsequent publication of ISIS-4, a large multi-center trial involving over 58,000 patients, showed not only the absence of significant reduction in arrhythmias or mortality with magnesium, but in fact a trend towards an increased risk of heart failure $[49,50]$, results that have since been further validated by publication of the MAGIC trial [51]. The early metaanalyses on intravenous magnesium were perhaps influ- 
enced by publication bias and the combination of data from several small randomized controlled trials [52].

There are many parallels between the intravenous magnesium story and our meta-analysis findings for acetylcysteine. The marked heterogeneity of findings across studies, and the finding of funnel plot asymmetry (indicating possible publication bias), ought to be viewed as strong cautionary points against making firm conclusions about the efficacy of acetylcysteine. And while it is true that acetylcysteine is inexpensive, easy to use and has a favorable side-effect profile, it is probably premature to conclude scientifically that it is definitely efficacious based on data published to date. Our firm conclusion based on this meta-analysis of published trails is that although the data seem quite promising, the efficacy of acetylcysteine has not been definitively proven.

To isolate potential sources of heterogeneity we performed a meta-regression analysis exploring several clinical and study quality factors. There was no evidence of association between effect size and baseline serum creatinine, volume of contrast media, or diabetes mellitus, all independently identified risk factors for development of contrast-induced nephropathy $[8,53]$. However, whether the angiographic procedure was performed electively or as emergency showed a significant relation with the size of the acetylcysteine effect. The need to perform emergency cardiac angiography is common in patients presenting with suspected acute coronary syndromes. Patients undergoing emergency coronary angiography have been shown to have increased mortality and poor long-term survival, independent of the development of contrast-induced nephropathy $[6,54]$.

Funnel plot asymmetry is often interpreted to indicate publication bias. However, it is important to consider that this asymmetry may also be due to other sources of bias that deserve further examination. In particular, fundamental disparities in study design, inconsistencies in methodological quality and differences in the definition of primary outcomes may have contributed to funnel plot asymmetry. Our meta-regression analysis explored the potential role of several study quality factors, and none were identified as statistically significant predictors of apparent acetylcysteine efficacy across trials. Nonetheless, it is quite possible that other unmeasured study quality factors may have contributed to biased results and accompanying funnel plot asymmetry.

Contrast-induced nephropathy continues to be an active subject matter for clinical investigation [55,56]. A definitive randomized clinical trial comparing fenoldopam, a selective type 1 dopamine receptor agonist, with placebo recently demonstrated no significant difference in the incidence of contrast-induced nephropathy or any secondary outcomes including 30 day mortality, need for dialysis, or re-hospitalization rates [56]. Another, recent randomized trial of 192 patients undergoing intravascular angiography compared prophylactic acetylcysteine with fenoldopam [57]. The results demonstrated a 9.6\% absolute risk reduction in patients randomized to acetylcysteine $(4.1 \%$ vs $13.7 \%$, respectively). Although the authors conclude that acetylcysteine is superior to fenoldopam for prevention of contrast-induced nephropathy, there was notably no significant difference in serum creatinine at $48 \mathrm{~h}$. Of interest, in subgroup analysis, the authors speculate that patients with low ejection fractions $(<40 \%)$ may attain additional benefit with acetylcysteine.

\section{Conclusion}

All of the above leads us to conclude that while acetylcysteine appears to be safe and inexpensive, its efficacy for the prevention of contrast-induced nephropathy remains unproven. The results of the trials that we reviewed to date should be viewed as early promising evidence of benefit, and suggest that it is now perhaps reasonable to use acetylcysteine in routine care because of its relative ease of use and safety. However, its true efficacy will remain uncertain unless a definitive well-designed multi-center trial is performed. Such a clinical trial will be most relevant if it addresses a priori clinically meaningful endpoints of renal insufficiency, rather than surrogate endpoints based on changes in creatinine levels alone, and further considers stratification on hypothesized important subgroups that may benefit such as those with a low ejection fraction [58].

\section{Competing interests}

The authors declare that they have no competing interests.

\section{Authors' Contributions}

SMB developed the study protocol, conducted literature search, screened articles for eligibility, extracted data, analyzed data, interpreted results, wrote and revised the manuscript. WAG contributed to protocol development, screened articles for eligibility, extracted data, analyzed data, interpreted results, and provided critique of successive drafts of the manuscript. Both authors read and approved the final manuscript.

\section{Acknowledgements}

Dr. Ghali is supported by a Government of Canada Research Chair in Health Services Research, and by a Health Scholar Award from the Alberta Heritage Foundation for Medical Research.

\section{References}

I. Hou SH, Bushinsky DA, Wish JB, Cohen JJ, Harrington JT: Hospitalacquired renal insufficiency: $A$ prospective study. American Journal of Medicine 1983, 74:243-248. 
2. Shusterman N, Strom B, Murray T: Risk factors and outcome of hospital-acquired acute renal failure. American Journal of Medicine 1987, 83:65-71.

3. Murphy SW, Barrett BJ, Parfrey PS: Contrast nephropathy. Journal of the American Society of Nephrology 2000, I I: I77-182.

4. Levy EM, Viscoli CM, Horwitz RI: The effect of acute renal failure on mortality. JAMA 1996, 275: | 489-I494.

5. Powe N, Moore R, Steinberg E: Adverse reactions to contrast media: Factors that determine the cost of treatment. $A J R$ 1993, 161:1089-1095.

6. Gruberg L, Mintz GS, Mehran R, Dangas G, Lansky AJ, Kent KM, Pichard A, Satler LF, Leon MB: The prognostic implications of further renal function deterioration within $48 \mathrm{~h}$ of interventional coronary procedures in patients with pre-existent chronic renal insufficiency. Journal of the American College of Cardiology 2000, 36: I542-1548.

7. Davidson C, Hlatky M, Morris K: Cardiovascular and renal toxicity of a nonionic radiocontrast agent after cardiac catheterization. Annals of Internal Medicine 1989, I I 0: I 19.

8. Rich MW, Crecelius CA: Incidence, risk factors, and clinical course of acute renal insufficiency after cardiac catheterization in patients $\mathbf{7 0}$ years of age or older. Archives of Internal Medicine 1990, 150:1237-1242.

9. Bakris GZ, Lass N, Gager A: Radiocontrast medium-induced declines in renal function: $A$ role for oxygen free radicals. $A m$ J Physiol 1990, 258:FII5-FI20.

10. Bakris G, Lass N, Glock D: Renal hemodynamics in radiocontrast medium-induced renal dysfunction. Kidney International 1999, 56:206-210.

II. Heyman S, Reichman J, Brezis M: Pathophysiology of radiocontrast nephropathy: a role for medullary hypoxia. Invest Radiol 1999, 34:685-691.

12. DiMari J, Megyesi J, Udvarhelyi N, Price P, Davis R, Safirstein R: Nacetyl cysteine ameliorates ischemic renal failure. Am J Physiol 1997, 272:F292-F298.

13. Lefebvre $C$, Clarke M: Identifying randomized trials. In Systematic Reviews in Health Care Second edition. Edited by: Egger M, Smith G, Altman D. London: BMJ Publications; 2001:69-86.

14. Robinson K, Hinegardner P, Lansing P: Development of an optimal search strategy for the retrieval of controlled trials using Pubmed. In Proc 6th Int Cochrane Collouium 1998. poster BI3

15. Dickersin K, Scherer R, Lefebvre C: Identifying relevant studies for systematic reviews. BMJ 1994, 309:|286- 229 .

16. Jadad A, Moore R, Carrol D, Jenkinson C, Reynolds J, Gavaghan D, McQuay $\mathrm{H}$ : Assessing the quality of reports of randomized clinical trials: Is blinding necessary? Control Clin Trials 1996, 17:1-12.

17. Juni P, Altman D, Egger M: Assessing the quality of randomised controlled trials. In: Systematic Reviews in Health Care Second edition. Edited by: Egger M, Smith G, Altman D. London: BMJ Publishing; 200I:87-108.

18. Shea B, Dube C, Moher D: Assessing the quality of reports of systematic reviews: the QUOROM statement compared to other tools. In: Systematic Reviews in Health Care Second edition. Edited by: Egger M, Smith G, Altman D. London: BMJ Publishing; 200I:122-139.

19. Thompson $\mathrm{S}$ : Why sources of heterogeneity in meta-analysis should be investigated. BMJ 1994, 309:|35|-| 355.

20. DerSimonian R, Laird N: Meta-analysis in clinical trials. Control Clin Trials 1986, 7:177-188.

21. Senn S, Walter S, Olkin I: Odds ratios revisited. Evidence-Based Med 1998, 3:71.

22. Thompson S, Sharp S: Explaining heterogeneity in meta-analysis: a comparison of methods. Stat Med 1999, 18:2693-2708.

23. Dear K, Begg C: An approach to assessing publication bias prior to performing a meta-analysis. Stat Sci 1992, 7:237-245

24. Egger M, Davey S, Schneider M, Minder C: Bias in meta-analysis detected by a simple, graphical test. BMJ 1997, 3 I 5:629-634.

25. Allaqaband S, Tumuluri R, Malik A: Prospective randomized study of $\mathrm{N}$-acetylcysteine, fenoldopam, and saline for prevention of radiocontrast-induced nephropathy. Catheter Cardiovasc Interv 2002, 57:279-283.

26. Baker C, Wragg A, Kuna S, DePalma R, Baker L: A rapid protocol for the prevention of contrast-inducted renal dysfunction: The RAPPID Study. Journal of the American College of Cardiology 2003, 4I:2 I |4-2I I8.
27. Briguori C, Manganelli F, Scarpato P, Elia PP, Golia B: Acetylcysteine and contrast agent-associated nephrotoxicity. Journal of the American College of Cardiology 2002, 40:298-303.

28. Diaz-Sandoval LJ, Kosowsky BD, Losordo DW: Acetylcysteine to prevent angiography-related renal tissue injury (The APART Trial). American Journal of Cardiology 2002, 89:356-358.

29. Durham JD, Caputo C, Dokko J, Zaharakis T, Pahlavan M: A randomized controlled trial of $\mathbf{N}$-acetylcysteine to prevent contrast nephropathy in cardiac angiography. Kidney International 2002, 62:2202-2207.

30. Efrati S, Dishy V, Averbukh M, Blatt A, Krakover R, Weisgarten J, Morrow J, Stein M, Golik A: The effect of $\mathbf{N}$-acetylcysteine on renal function, nitric oxide, and oxidative stress after angiography. Kidney International 2003, 64:2182-2187.

3I. Fung J, Szeto C, Chan W, Kum L, Chan A, Wong J, Wu E, Yip G, Chan J, Yu C, Woo K, Sanderson J: Effect of $\mathbf{N}$-acetylcysteine for prevention of contrast nephropathy in patients with moderate to severe renal insufficiency: a randomized trial. American Journal of Kidney Diseases 2004, 43:80 I-808.

32. Goldenberg I, Schechter M, Matetzki S, Jonas M, Adam M, Pres H, Elian D, Agranat O, Schwammenthal E, Guetta V: Oral acetylcysteine as an adjunct to saline hydration for prevention of contrast-induced nephropathy following coronary angiography. Eur Heart J 2004, 25:2 I 2-2 I8.

33. Kay J, Chow WH, Chan TM, Lo SK, Kwok OH, Yip A: Acetylcysteine for prevention of acute deterioration of renal function following elective coronary angiography and intervention: a randomized controlled trial. JAMA 2003, 289:553-558.

34. Kefer J, Hanet C, Boitte S, Wilmotte L, De Kock M: Acetylcysteine, coronary procedure and prevention of contrast-induced worsening of renal function: which benefit for which patients? Acta Cardiol 2003, 58:555-560.

35. MacNeill B, Harding S, Bazari H, Patton K, Colon-Hernadez P, de Joseph D, Jang I: Prophylaxis of contrast-induced nephropathy in patients undergoing coronary angiography. Catheter Cardiovasc Interv 2003, 60:458-46I.

36. Oldemeyer JB, Biddle P, Wurdeman R, Mooss A, Cichowski E, Hilleman D: Acetylcysteine in the prevention of contrast-induced nephropathy after coronary angiography. Am J Heart 2003, 146:e23.

37. Shyu K-G, Cheng J-J, Kuan P: Acetylcysteine protects against acute renal damage in patients with abnormal renal function undergoing a coronary procedure. Journal of the American College of Cardiology 2002, 40: I383-I388.

38. Vallero A, Cesano G, Pozzato M, Garbo R, Minelli M, Quarello F, Formica M: Contrast nephropathy in cardiac procedures: No advantages with prophylactic use of $\mathbf{N}$-acetylcysteine (NAC). Giornale Italiano di Nefrologia 2002, 19:529-533.

39. Cook D, Guyatt G, Ryan G, Clifton J, Buckingham L, Willan A, Mcllroy $W$, Oxman A: Should unpublished data be included in metaanalyses? Current convictions and controversies. JAMA 1993, 269:2749-2753.

40. Birck R, Krzossok S, Makowetz F, Schnulle P, van der Woude F, Braun C: Acetylcysteine for prevention of contrast nephropathy: meta-analysis. Lancet 2003, 362:598-603.

4I. Isenbarger D, Kent S, O'Malley P: Meta-analysis of randomized clinical trials on the usefulness of acetycysteine for prevention of contrast nephropathy. Am J Card 2003, 92: |454- 4458.

42. Alonso A, Lau J, Jaber B, Weintraub A, Sarnak M: Prevention of radiocontrast nephropathy with $\mathrm{N}$-acetylcysteine in patients with chronic kidney disease: a meta-analysis of randomized, controlled trials. American Journal of Kidney Diseases 2004, 43:I-9.

43. Faddy S: Significant statistical heterogeneity in a meta-analysis of the usefulness of acetylcysteine for prevention of contrast nephropathy. Am J Cardiol 2004, 94:4I4.

44. Pannu N, Manns B, Lee H, Tonelli M: Systematic review of the impact of $\mathbf{N}$-acetylcysteine on contrast nephropathy. Kidney International 2004, 65: I 366-1374.

45. Kshirsagar A, Poole C, Mottl A, Shoham D, Francechini N, Tudor G, Agrawal M: $\mathbf{N}$-acetylcysteine for the prevention of radiocontrast induced nephropathy: A meta-analysis of prospective controlled trials. Journal of the American Society of Nephrology 2004, I5:76I-769. 
46. Teo KK, Yusuf S, Collins R, Held PH, Peto R: Effects of intravenous magnesium in suspected acute myocardial infarction: overview of randomized trials. BMJ 1991, 303:|499-I503.

47. Horner S: Efficacy of intravenous magnesium in acute myocardial infarction in reducing arrhythmias and mortality: Meta-analysis of magnesium in acute myocardial infarction. Circulation 1992, 86:774-779.

48. Yusuf S, Koon T, Woods K: Intravenous magnesium in acute myocardial infarction. An effective, safe, simple, and inexpensive intervention. Circulation 1993, 87:2043-2046.

49. Group I-FISoISC: ISIS-4: A randomized factorial trial assessing early oral captopril, oral mononitrate, and intravenous magnesium sulphate in 58050 patients with suspected acute myocardial infarction. Lancet 1995, 345:669-685.

50. Baxter G, Sumeray M, Walker J: Infarct size and magnesium: insights into LIMIT-2 and ISI-4 from experimental studies. Lancet 1996, 348: |424-| 426.

51. Investigators TMiCMT: Early administration of intravenous magnesium to high-risk patients with acute myocardial infarction in the Magnesium in Coronaries (MAGIC) Trial: a randomized controlled trial. Lancet 2002, 360: I I89- I 196.

52. Egger M, Davey Smith G: Misleading meta-analysis. BMJ I995, 3 I 0:352-354.

53. Guitterez N, Diaz A, Timmis G, O'Neill WW, Stevens MA, Sandberg $K$, McCullough PA: Determinants of serum creatinine trajectory in acute contrast nephropathy. J Interv Cardiol 2002 I 5:349-354.

54. Hemmelgarn BR, Ghali WA, Quan H, Brant R, Norris CM: Poor long-term survival after coronary angiography in patients with renal insufficiency. American Journal of Kidney Diseases 200 I, 37:64-72.

55. Merten G, Burgess W, Gray L, Holleman J, Roush T, Kowalchuk G Bersin R, Moore A, Simonton C, Rittase R, Norton H, Kennedy T: Prevention of contrast-induced nephropathy with sodium bicarbonate. JAMA 2004, 29 I:2328-2334.

56. Stone G, Mc Cullough P, Tumlin J, Lepor N, Madyoon H, Murray $P$ Wang A, Chu A, Schaer G, Stevens M, Wilensky R, O'Neill W: Fenoldopam mesylate for the prevention of contrastinduced nephropathy: A randomized controlled trial. JAMA 2003, 290:2284-2291.

57. Briguori C, Colombo A, Airoldi F, Violante A, Castelli A, Balestrieri P, Elia PP, Golia B, Lepore S, Riviezzo G, Scarpato P, Librera M, Focaccio $A$, Ricciardelli $B$ : $\mathbf{N}$-acetylcysteine versus fenoldopam mesylate to prevent contrast agent-associated nephrotoxicity. Journal of the American College of Cardiology 2004, 44:762-765

58. Bagshaw S, Culleton B: Acetylcysteine for the prevention of contrast nephropathy. JAMA 2003, 289:2795.

\section{Pre-publication history}

The pre-publication history for this paper can be accessed here:

http://www.biomedcentral.com/1741-7015/2/38/prepub
Publish with Biomed Central and every scientist can read your work free of charge

"BioMed Central will be the most significant development for disseminating the results of biomedical research in our lifetime. "

Sir Paul Nurse, Cancer Research UK

Your research papers will be:

- available free of charge to the entire biomedical community

- peer reviewed and published immediately upon acceptance

- cited in PubMed and archived on PubMed Central

- yours - you keep the copyright

Submit your manuscript here:

http://www.biomedcentral.com/info/publishing_adv.asp
BioMedcentral 